ISSN electrónico: 2445-1355

DOI: https://doi.org/10.14201/fj2021615363

\title{
APLICACIÓN DE LA TÉCNICA LAMP PARA LA DETECCIÓN DE LOA LOA Y MANSONELLA PERSTANS
}

\section{Application of the LAMP Technique for the Detection of Loa Loa and Mansonella perstans}

Gloria GALLEGO; Begoña FEBRER-SENDRA; Beatriz CREGO-VICENTE; Juan GARCÍA-BERNALT DIEGO; Pedro FERNÁNDEZ-SOTO

Departamento Biología Animal, Parasitología, Ecología, Edafología y Química Agrícola. Facultad de Farmacia. Universidad de Salamanca. Licenciado Méndez Nieto. 37001 Salamanca

Correo-e: gloriagh_97@usal.es

RESUMEN: Las filariosis son enfermedades endémicas de regiones tropicales ocasionadas por nematodos filiformes trasmitidos por la picadura de insectos. Producen elevada morbilidad. La loaosis (Loa loa) y la mansonelosis (Mansonella perstans) afectan globalmente a $10 \mathrm{y}$ 100 millones de personas, respectivamente. El diagnóstico de certeza es parasitológico, pero no detecta infecciones precoces o bajas microfilaremias y hay que considerar la periodicidad de las microfilarias en sangre. Las técnicas moleculares, como la PCR, tienen gran sensibilidad y especificidad, pero son caras, técnicamente complejas y requieren infraestructura no disponible en zonas endémicas de escasos recursos. La tecnología LAMP (loop-mediated isothermal amplification) presenta ventajas sobre la PCR como mayor rapidez, escaso equipamiento, más tolerante a inhibidores y los resultados pueden observarse colorimétricamente. En este trabajo se aplica y valora la tecnología LAMP para la detección de ADN de Loa loa y $M$. perstans en 22 muestras de sangre almacenadas en papel de filtro de individuos residentes en Guinea Ecuatorial. Las muestras se analizaron microscópicamente, mediante qPCR y LAMP. Los métodos moleculares 
resultaron más sensibles que la microscopía. El LAMP resultó más sensible que la qPCR para la detección de ADN de Loa loa y M. perstans.

Palabras clave: filariosis; Loa loa; Mansonella perstans; qPCR; LAMP.

ABSTRACT: Filariases are endemic diseases of tropical regions caused by filiform nematodes transmitted by insect bites. They produce high morbidity. Loiasis (Loa loa) and mansonellosis (Mansonella perstans) globally affect 10 and 100 million people, respectively. The diagnosis of certainty is parasitological, but it does not detect early infections or low microfilaeremia, and it is necessary to consider the periodicity of microphilaremias in blood. Molecular techniques, such as PCR, have great sensitivity and specificity, but they are expensive, technically complex and require infrastructure not available in endemic areas of scarce resources. LAMP technology (loop-mediated isothermal amplification) has advantages over PCR as faster, scarce equipment, more tolerant of inhibitors and the results can be observed colorimetrically. In this work, LAMP technology is applied and evaluated for the detection of DNA from Loa loa and M. perstans in 22 blood samples stored on filter paper from individuals living in Equatorial Guinea. The samples were analyzed microscopically, using qPCR and LAMP. The molecular methods were more sensitive than microscopy. LAMP resulted more sensitive than qPCR for the detection of DNA from Loa loa and M. perstans.

Keywords: filariasis; Loa loa; Mansonella perstans; qPCR; LAMP.

\section{INTRODUCCIÓN}

Las filariosis son un grupo de enfermedades que afectan al hombre (en algunos casos a animales) ocasionadas por nematodos filiformes («filarias») de la familia Filarioidea, transmitidas por la picadura de insectos vectores. Son endémicas de áreas tropicales y constituyen un importante problema de salud pública en países subdesarrollados. Su mortalidad es baja, pero las manifestaciones crónicas producen elevada morbilidad con consecuencias psíquicas, sociales y médicas de gran repercusión económica en zonas endémicas (Díaz-Menéndez et al., 2011). Se clasifican en «filariosis clásicas» y otras filariosis (dracunculiasis y dirofilariosis) de menor entidad. Las filariosis clásicas se clasifican según la localización del agente causal en filariosis linfáticas, cutáneo/oculares, cutáneas e inespecíficas (Pérez-Arellano et al., 2010). 
Las filarias que afectan al hombre tienen un ciclo biológico similar. La fase parasitaria responsable de las manifestaciones clínicas es el helminto adulto en Wuchereria bancrofti, Brugia malayi y Brugia timori, la microfilaria en Onchocerca volvulus, o ambas fases en Loa loa y Mansonella spp.(Díaz-Menéndez et al., 2011).

La Organización Mundial de la Salud (OMS) incluye a las filariosis linfáticas y a la oncocercosis entre las 20 enfermedades tropicales desatendidas (NTD, del inglés Neglected Tropical Diseases). Presentan elevada prevalencia a nivel mundial. La loaosis y la mansonelosis no están entre las 20 NTD, aunque la loaosis afecta a más de 10 millones de personas y la mansonelosis cutánea por M. perstans infecta a 100 millones de personas (Whittaker et al., 2018; Ta-Tang et al., 2018). La loaosis es generalmente asintomática, con eosinofilia frecuente, ocasionando angioedemas (edemas de Calabar) y la migración del adulto por el tejido subconjuntival (Simonsen et al., 2014). La mansonelosis por M. perstans, menos conocida, produce edemas subcutáneos tipo Calabar, exantemas, dolor abdominal y eosinofilia (Simonsen et al., 2011). Es frecuente la infección simultánea con distintas filarias (Akue et al., 2011).

En ausencia de vacunas, el tratamiento y los programas de control y erradicación de filariosis de la OMS se basan en la quimioprofilaxis masiva mediante MDA (Mass Drug Administration) y aliviando la morbilidad. Se fundamentan en tres fármacos: dietilcarbamacina (DEC), activo frente a microfilarias y adultos, ivermectina (IV) y albendazol (ABZ), ambos activos solo frente a adultos.

Individualmente, el fármaco de elección para la loaosis y $M$. streptocerca es DEC, para $M$. perstans es ABZ y para $M$. ozzardi es IV. Los efectos secundarios son raros, excepto cuando hay infección por varias filarias (entre ellas, Loa loa) y la microfilaremia es alta, produciéndose la muerte de filarias provocando deterioro neurológico progresivo y encefalopatías (Taylor et al., 2010). La loaosis ha representado un impedimento a la efectividad de los programas de MDA debido a los efectos adversos de IV en pacientes con alta carga parasitaria (Whittaker $e t$ al., 2018).

Así, el diagnóstico de las filariosis es fundamental para un tratamiento seguro y efectivo. El método de referencia es la detección de microfilarias en sangre o piel mediante microscopía o de adultos en órganos o tejidos (Loa loa en tejido conjuntivo). Tiene alta especificidad, pero no detecta infecciones precoces o bajas microfilaremias y hay que considerar la periodicidad de las microfilarias para obtener la sangre. La serología tiene valor limitado y presenta reacciones cruzadas con otros helmintos (Díaz-Menéndez et al., 2011). Las técnicas moleculares como la PCR tienen gran sensibilidad y especificidad, permitiendo diferenciar filarias en bajas parasitemias (Ta-Tang et al., 2010; Ta-Tang et al., 2018). Sin embargo, requieren infraestructura de alto coste y personal especializado que impiden su uso en zonas endémicas de escasos recursos. Una alternativa es la técnica de 
amplificación isotérmica de ácidos nucleicos tipo LAMP (Loop-mediated isothermal amplification) descrita por Notomi et al. (2000). Se basa en el principio de síntesis de ADN por desplazamiento de cadena, realizado por una Bst polimerasa (Bacillus stearothermophylus) con elevada actividad de desplazamiento a temperatura constante de $60-65^{\circ} \mathrm{C}$ y un sistema de 4 o 6 cebadores: dos internos, Forward Inner Primer (FIP) y Backward Inner Primer (BIP); dos externos (F3 y B3), y, opcionalmente, 2 cebadores bucle o loop primers (FLP y FLB), para acelerar la reacción por la unión a sitios no accesibles a los cebadores internos. En total, se reconocen 6 a 8 secuencias distintas en el ADN diana, obteniéndose gran especificidad y sensibilidad. Las ventajas del LAMP respecto a otros métodos moleculares son la rapidez, no requerir aparataje sofisticado (solo requiere un termobloque), es más tolerable a inhibidores de muestras y los resultados pueden visualizarse por cambio colorimétrico añadiendo un colorante fluorescente (Li Y et al., 2017).

\section{Hipótesis}

El grupo de investigación «Enfermedades Infecciosas y Tropicales» (e-INTRO) del IBSAL-CIETUS tiene amplia experiencia en el desarrollo de métodos LAMP para la detección de diferentes parásitos, incluyendo un LAMP para la detección sensible y específica de Loa loa (Fernández-Soto et al., 2014). Este LAMP solo ha sido evaluado con muestras artificiales preparadas en el laboratorio. Sería de gran interés evaluar su funcionamiento en muestras clínicas de individuos residentes en zona endémica de loaosis y también evaluar un LAMP para la detección de $M$. perstans (Poole et al., 2019), pues su diagnóstico pasa frecuentemente inadvertido.

\section{3. Овjetivos}

\subsection{Objetivo general}

Aplicar y valorar la tecnología LAMP para la detección de ADN de Loa loa y $M$. perstans en muestras de sangre de individuos residentes en zona endémica de loaosis y mansonelosis de Guinea Ecuatorial.

\subsection{Objetivos específicos}

- Realizar y comprobar el funcionamiento de la técnica LAMP para la detección de Loa loa y M. perstans.

- Analizar y evaluar la técnica LAMP para la detección de Loa loa y M. perstans en muestras de individuos residentes en zona endémica. 


\section{Materiales Y Métodos}

\subsection{Muestras y zona de estudio}

Se seleccionaron 22 muestras sanguíneas de 350 recolectadas de población residente en el distrito de Bata, Guinea Ecuatorial, como parte del estudio realizado por el Centro Nacional de Medicina Tropical (CNMT) del Instituto de Salud Carlos III (ISCIII) para conocer la prevalencia de diferentes parasitosis.

\subsection{Obtención y análisis de las muestras}

La sangre se recolectó para el análisis microscópico de diferentes parásitos, incluyendo filarias. Un volumen de la extracción se almacenó en papel de filtro (Whatman n..$^{\circ}$ ) y se secó al aire (manchoteca). En el CNMT se extrajo el ADN de cada papel de filtro mediante saponina-Chelex y se analizó mediante PCR a tiempo real (qPCR) para la detección específica de Loa loa y M. perstans según el protocolo descrito por Ta-Tang et al. (2010). Alícuotas del ADN se enviaron al CIETUS para su estudio y comparación con la técnica LAMP.

\subsection{Preparación de $A D N$ de Loa loa y M. perstans}

Se utilizó ADN disponible en la colección del CIETUS como control positivo en las reacciones de LAMP. Se amplificó utilizando el kit comercial Genomiphi ${ }^{\mathrm{TM}} \mathrm{V} 2$ (GE Healthcare). Se midió la concentración en un Nanodrop ND-100, se hicieron diluciones de trabajo $(0,5 \mathrm{ng} / \mu \mathrm{L}$ y $5 \mathrm{ng} / \mu \mathrm{L})$ y se almacenaron a -20 ${ }^{\circ} \mathrm{C}$ hasta su uso.

\subsection{LAMP para la detección de Loa loa y M. perstans}

El LAMP para la detección de Loa loa se realizó utilizando el método de Fernández-Soto et al. (2014) y para M. perstans, según lo descrito por Poole et al. (2019). Las reacciones se realizaron en un termobloque a $60^{\circ} \mathrm{C}$ durante $45 \mathrm{~min}$, más 10 min a $80^{\circ} \mathrm{C}$ para inactivar la enzima. Como controles positivos en las reacciones de LAMP se utilizó ADN de Loa loa $(1 \mu \mathrm{L} ; 0.5 \mathrm{ng} / \mu \mathrm{L})$ o M. perstans $(2 \mu \mathrm{L} ; 5 \mathrm{ng} / \mu \mathrm{L})$.

\subsection{Detección de los productos de amplificación}

Se realizó colorimétricamente, añadiendo postamplificación a los tubos $2 \mu \mathrm{L}$ de SYBR ${ }^{\circledR}$ Green I (1:10; 10,000X; Invitrogen). Las muestras positivas viraron a verde y las negativas mantuvieron el color naranja original del colorante. El 
resultado se corroboró en electroforesis de agarosa al $1.5 \%$ (100 mL de TBE $0.5 \mathrm{X}, 1.5 \mathrm{~g}$ de agarosa y $5 \mu \mathrm{L}$ de Midori Green) a $60 \mathrm{v}$ para observar el patrón de bandas «en escalera» característico de la amplificación mediante LAMP. Los geles se fotografiaron y las imágenes se guardaron digitalmente para su edición.

\section{Resultados}

\subsection{Análisis parasitológico y molecular mediante qPCR}

Se realizaron en el CNMT. Seleccionamos 22 muestras con análisis parasitológico: 12 muestras negativas para Loa loa y M. perstans (n. ${ }^{\text {os }} 1-12$ ); 5 positivas para Loa loa y negativas para $M$. perstans (n. $\left.{ }^{\text {os }} 16-20\right)$; 3 positivas para $M$. perstans y negativas para Loa loa (n. $\left.{ }^{\text {os }} 13-15\right) ; 2$ muestras con coinfección (n. ${ }^{\text {os }} 21-22$ ) (Tabla 1).

TABLA 1. Resultados del análisis parasitológico por microscopía, qPCR y LAMP para la detección de Loa loa y Mansonella perstans. Se indica número de muestra y resultado positivo o negativo para cada una de las muestras analizada por cada técnica. La microscopía y qPCR se realizó en el CNMT del ISCIII

\begin{tabular}{c|c|c|c|c|c}
\hline & \multicolumn{2}{|c|}{ Microscopía } & \multicolumn{2}{c|}{ qPCR } & LAMP \\
\hline $\begin{array}{c}\text { N. } \\
\text { mustra }\end{array}$ & Loa loa & M. perstans & Loa Loa & M. perstans & Loa loa \\
\hline 1 & Negativo & Negativo & Negativo & Negativo & Negativo \\
\hline 2 & Negativo & Negativo & Negativo & Negativo & Negativo \\
\hline 3 & Negativo & Negativo & Negativo & Negativo & Negativo \\
\hline 4 & Negativo & Negativo & Negativo & Negativo & Negativo \\
\hline 5 & Negativo & Negativo & Negativo & Negativo & Negativo \\
\hline 6 & Negativo & Negativo & Negativo & Negativo & Negativo \\
\hline 7 & Negativo & Negativo & Negativo & Negativo & Negativo \\
\hline 8 & Negativo & Negativo & Negativo & Negativo & Negativo \\
\hline 9 & Negativo & Negativo & Negativo & Negativo & Negativo \\
\hline 10 & Negativo & Negativo & Negativo & Negativo & Negativo \\
\hline 11 & Negativo & Negativo & Negativo & Positivo & Negativo \\
\hline 12 & Negativo & Negativo & Negativo & Positivo & Negativo \\
\hline 13 & Negativo & Positivo & Negativo & Positivo & Negativo \\
\hline 14 & Negativo & Positivo & Negativo & Positivo & Negativo \\
\hline 15 & Negativo & Positivo & Negativo & Positivo & Negativo \\
\hline
\end{tabular}


GLORIA GALLEGO; BEGOÑA FEBRER-SENDRA; BEATRIZ CREGO-VICENTE;

JUAN GARCÍA-BERNALT DIEGO; PEDRO FERNÁNDEZ-SOTO

APLICACIÓN DE LA TÉCNICA LAMP PARA LA DETECCIÓN DE LOA LOA Y MANSONELLA PERSTANS

\begin{tabular}{c|c|c|c|c|c}
\hline & \multicolumn{2}{|c|}{ Microscopía } & \multicolumn{2}{c|}{ qPCR } & LAMP \\
\hline $\begin{array}{c}\text { N. } \\
\text { muestra }\end{array}$ & Loa loa & M. perstans & Loa Loa & M. perstans & Loa loa \\
\hline 16 & Positivo & Negativo & Positivo & Negativo & Positivo \\
\hline 17 & Positivo & Negativo & Positivo & Negativo & Positivo \\
\hline 18 & Positivo & Negativo & Positivo & Negativo & Positivo \\
\hline 19 & Positivo & Negativo & Positivo & Negativo & Positivo \\
\hline 20 & Positivo & Negativo & Positivo & Negativo & Positivo \\
\hline 21 & Positivo & Positivo & Negativo & Negativo & Positivo \\
\hline 22 & Positivo & Positivo & Positivo & Positivo & Positivo \\
\hline
\end{tabular}

5.2. Reacciones de LAMP para la detección de Loa loa y M. perstans

Los resultados del funcionamiento del LAMP en la amplificación del ADN de ambas filarias se muestran en la Figura 1.

A) LAMP-Loa loa

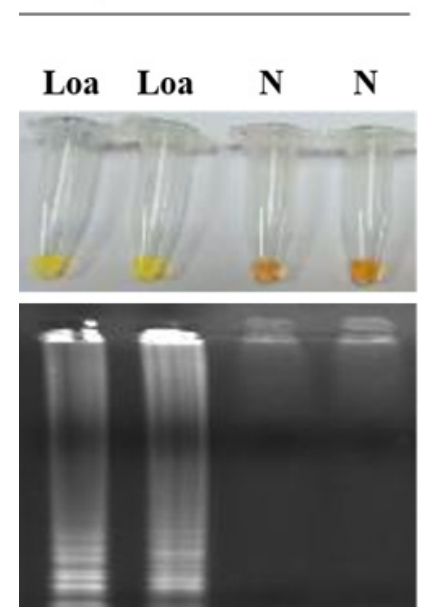

B) LAMP-M. perstans
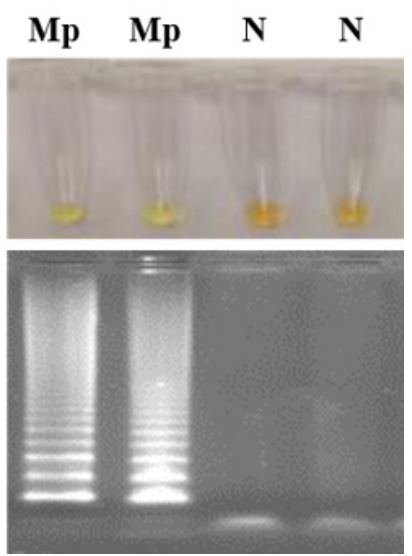

Figura 1. LAMP para la amplificación de Loa loa según Fernández-Soto et al. (2014) (A) y Mansonella perstans según Poole et al. (2019) (B). Establecimiento de la reacción con los juegos de cebadores correspondientes en cada caso y condiciones de 60 ${ }^{\circ} \mathrm{C}$ durante 60 min más $80^{\circ} \mathrm{C}$ durante 5-10 min. Loa, ADN de Loa loa; Mp, ADN de Mansonella perstans; N, controles negativos (no ADN). Arriba: resultados colorimétricos por adición postamplificación de SYBR Green I; abajo: patrón de bandas en electroforesis. 


\subsection{Análisis de las muestras mediante LAMP}

- LAMP para la detección de Loa loa

Todas las muestras parasitológicamente negativas para Loa loa resultaron negativas por LAMP (color naranja), incluyendo aquellas parasitológicamente positivas para $M$. perstans. En las 7 muestras parasitológicamente positivas para Loa loa se obtuvo amplificación mediante LAMP (color verde), incluyendo las 2 con coinfección con $M$. perstans (Figura 2A y Figura 3).

- LAMP para la detección de M. perstans

De 17 muestras negativas por microscopía (n. ${ }^{\text {os }} 1-12$ y 16-20), se obtuvieron resultados positivos por LAMP en 4 muestras $\left(n .{ }^{\circ} 1,7,11,12\right)$. Las 3 muestras parasitológicamente positivas para $M$. perstans resultaron positivas mediante LAMP (n. $\left.{ }^{\circ} 13-15\right)$; también las muestras con coinfección con Loa loa (n. ${ }^{\text {os }} 21$ y 22) (Figura $2 \mathrm{~B}$ y Figura 3 ).

A) Lamp-Loa loa
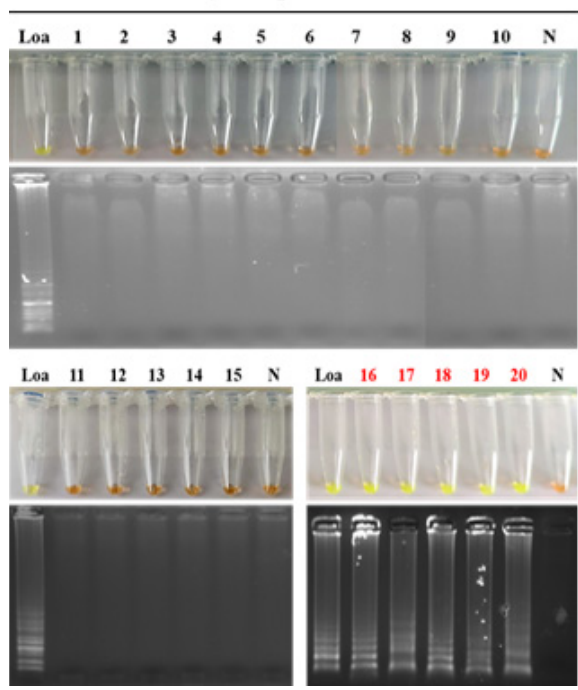

B) Lamp-M. perstans
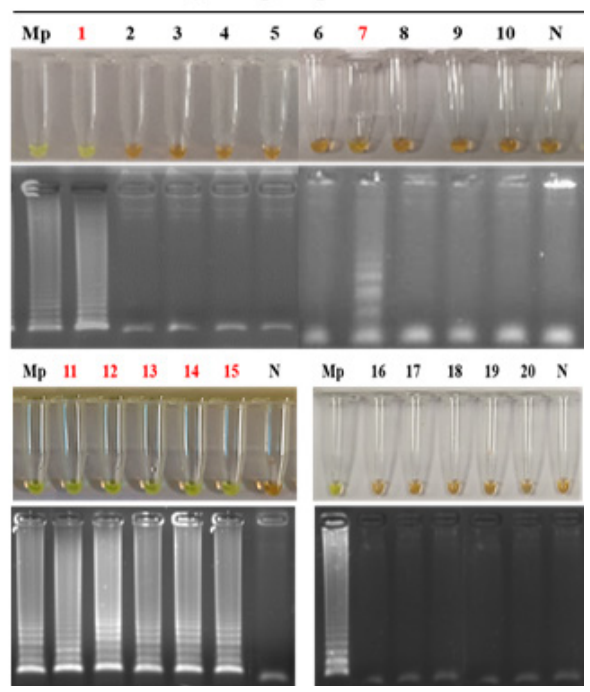

Figura 2. Análisis mediante LAMP para la detección de Loa loa según el método de Fernández-Soto et al. (2014) (A) y para la detección de Mansonella perstans según el método de Poole et al. (2019) (B) de las muestras 1-20 incluidas en el estudio. Loa, ADN de Loa loa (control positivo); Mp, ADN de M. perstans (control positivo); N, control negativo (agua ultrapura, no ADN); 1-20, muestras analizadas. Arriba: resultados colorimétricos al añadir postamplificación SYBR Green I; abajo: electroforesis de agarosa mostrando el patrón de bandas típico en escalera. 
A. Loa loa

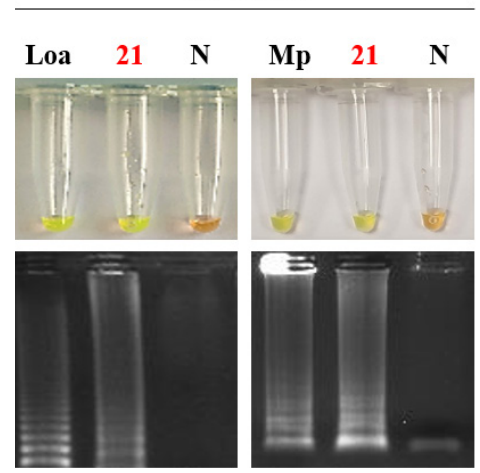

B. M. perstans

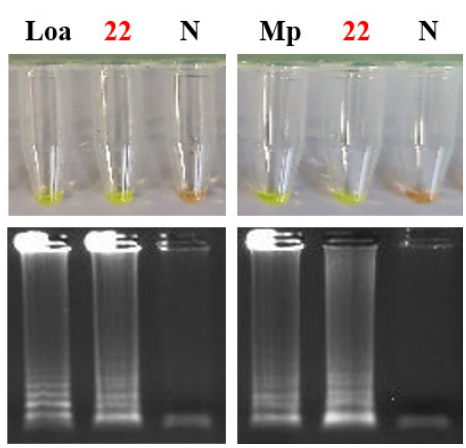

Figura 3. Análisis mediante LAMP para la detección de Loa loa según el método de Fernández-Soto et al. (2014) (A) y Mansonella perstans según el método de Poole et al. (2019) (B) de las muestras 21 y 22 que resultaron microscópicamente positivas para ambos parásitos. Loa, ADN de Loa loa (control positivo); $\mathrm{Mp}, \mathrm{ADN}$ de $M$. perstans (control positivo); $\mathrm{N}$, controles negativos (agua ultrapura, no ADN); 21 y 22 (en rojo), muestras microscópicamente positivas para ambos parásitos. Arriba: resultados colorimétricos al añadir postamplificación SYBR Green I; abajo: electroforesis de agarosa mostrando el patrón de bandas típico en escalera.

\section{Discusión}

Aunque exista sospecha clínica y eosinofilia, el diagnóstico de certeza de las filariosis es la detección microscópica de filarias. Aunque es específica, presenta baja sensibilidad y con problemas de diagnóstico en individuos amicrofilarémicos. Los métodos moleculares (PCR) han supuesto avances en sensibilidad, especificidad y rapidez de diagnóstico, pero son técnicamente complejos, de elevado coste y difícilmente aplicables en países subdesarrollados. Para solventar estos problemas, la tecnología LAMP es una excelente alternativa a la PCR (Mori y Notomi, 2020). En este trabajo utilizamos el LAMP para detectar ADN de Loa loa (Fernández-Soto et al., 2014) y ADN de M. perstans (Poole et al., 2019) evaluando su eficacia en el diagnóstico de 22 muestras clínicas seleccionadas de un estudio realizado en Guinea Ecuatorial. El LAMP de Loa loa resultó positivo en las 5 muestras confirmadas parasitológicamente, coincidiendo con los resultados de qPCR (n. $\left.{ }^{\text {os }} 16-20\right)$. Una sensibilidad equiparable en ambas técnicas ha sido observada previamente en análisis de muestras clínicas de residentes de Camerún (Drame et al., 2014). El LAMP de M. perstans resultó positivo en las 3 muestras confirmadas parasitológicamente, coincidiendo también con los resultados de 
qPCR (n. os 13-15). Además, 2 de las muestras negativas microscópicamente resultaron positivas por qPCR y por LAMP (n. ${ }^{\text {os }} 11$ y 12 ), demostrando mayor sensibilidad en ambas pruebas moleculares, aunque el LAMP detectó $M$. perstans en 2 muestras negativas más (n. ${ }^{\text {os }} 1$ y 7 ), evidenciando una mayor sensibilidad. Recientemente, se ha comprobado mayor sensibilidad del LAMP frente a la PCR en la detección de ADN de $M$. perstans en el análisis de muestras sanguíneas de pacientes e insectos vectores (Poole et al., 2019). En el estudio también incluimos dos muestras con infección por ambas filarias (n. ${ }^{\text {os }} 21$ y 22), pues en el oeste y centro de África son endémicas y es frecuente la infección con diferentes filarias (Akue et al., 2011). De nuevo, los métodos LAMP demostraron mayor sensibilidad en la detección de las dos especies respecto a la qPCR, que solo detectó el ADN de los parásitos en una de ellas (n. $\left.{ }^{\circ} 22\right)$. En nuestro trabajo hemos comprobado la mayor sensibilidad de los métodos LAMP respecto a la qPCR para la detección de Loa loa y $M$. perstans. Nuestro LAMP de Loa loa es la primera vez que se evalúa con muestras clínicas, obteniéndose un excelente resultado. Además, se ha utilizado ADN extraído mediante el método saponina-Chelex de muestras sanguíneas almacenadas en papel de filtro. El uso de protocolos rápidos, simples y más económicos que los métodos comerciales de extracción de ADN han sido utilizados con éxito en otros trabajos para la detección mediante LAMP de Loa loa en muestras clínicas (Drame et al., 2014). Aunque es necesario el análisis de mayor número de muestras, la eficacia demostrada del LAMP sobre la qPCR y la posibilidad de utilizar métodos simples de extracción del ADN de muestras hacen de la técnica LAMP una herramienta molecular sensible, específica y fácil de interpretar, potencialmente útil en el diagnóstico de la loaosis y mansonelosis en zonas endémicas de escasos recursos.

\section{Conclusiones}

Primera. El LAMP para la detección de Loa loa ha resultado eficaz en la detección del ADN del parásito obtenido mediante saponina-Chelex a partir de muestras de sangre almacenadas en papel de filtro.

Segunda. Se ha comprobado la eficacia descrita recientemente del LAMP desarrollado para la detección de Mansonella perstans en muestras clínicas.

\section{Bibliografía}

Akue JP, Nkoghe D, Padilla C, Moussavou G, Moukana H, Mbou RA et al. Epidemiology of Concomitant Infection Due to Loa loa and Mansonella perstans in Gabon. PLoS Negl Trop Dis. 2011; 5(10). 
Díaz-Menéndez M, Norman F, Monge-Maillo B, Pérez-Molina JA, López-Vélez R. Las filariasis en la práctica clínica. Enferm Infecc Microbiol Clin. 2011;29(Supl 5):27-37.

Drame PM, Fink DL, Kamgno J, Herrick JA, Nutman TB. Loop-mediated isothermal amplification for rapid and semiquantitative detection of Loa loa infection. J Clin Microbiol. 2014; 52(6):2071-2077.

Fernández-Soto P, Mvoulouga P, Akue JP, Abán J, Santiago B, Sánchez M. et al. Development of a Highly Sensitive Loop-Mediated Isothermal Amplification (LAMP) Method for the Detection of Loa loa. PLos ONE. 2014; 9(4).

Li Y, Fan P, Zhou S, Zhang L. Loop-mediated isothermal amplification (LAMP): A novel rapid detection platform for pathogens. Microb Pathog. 2017; 107:54-61.

Mori Y, Notomi T. Loop-mediated isothermal amplification (LAMP): Expansion of its practical application as a tool to achieve universal health coverage. J Infect Chemother. 2020; 26(1):13-17.

Notomi T, Okayama H, Masubuchi H, Yonekawa T, Watanabe K, Amino N et al. Loop-mediated isothermal amplification of DNA. Nucleic Acids Res. 2000; 28(12).

Pérez-Arellano JL, Carranza-Rodríguez C, Vieira-Lista C, Muro A. Nematodosis I: filariosis. Medicine. 2010; 10(55):3729-3738.

Poole C, Sinha A, Ettwiller L, Apone L, McKay K, Panchapakesa V et al. In Silico Identification of Novel Biomarkers and Development of New Rapid Diagnostic Tests for the Filarial Parasites Mansonella perstans and Mansonella ozzardi. Sci rep. 2019; 9(1).

Simonsen PE, Fischer PU, Hoerauf A, Weil GJ. The filariasis. En: Farrar J, Hotez PJ, Junghanss T, Kang G, Lalloo D, White NJ, editores. Manson's Tropical Diseases. 23. ${ }^{a}$ ed. London: Saunders; 2014. pp. 737-765.

Simonsen PE, Onapa AW, Asio SM. Mansonella perstans filariasis in Africa. Acta Trop. 2011; 120 (Suppl 1):S109-S120.

Ta-Tang TH, Crainey JL, Post RJ, Luz SL, Rubio JM. Mansonellosis: current perspectives. Rep Trop Med. 2018; 9:9-24.

Ta-Tang TH, López-Vélez R, Lanza M, Shelley AJ, Rubio JM, Luz SL. Nested PCR to detect and distinguish the sympatric filarial species Onchocerca volvulus, Mansonella ozzardi and Mansonella perstans in the Amazon Region. Men Inst Oswaldo Cruz. 2010; 105(6):823-828.

Taylor MJ, Hoerauf A, Bockarie M. Lymphatic filariasis and onchocerciasis. Lancet. 2010; 376(9747):1175-1185.

Whittaker C, Walker M, Pion SDS, Chesnais CB, Boussinesq M, Basáñez MG. The Population Biology and Transmission Dynamics of Loa loa. Trends Parasitol. 2018; 34(4):335-349. 
